\title{
Identifiabilité et activation référentielles dans les titres de presse
}

\author{
Salles, Mathilde \\ Normandie Université, UCBN \\ CRISCO, EA 4255 \\ mathilde.salles@unicaen.fr
}

L'objet de cette communication est de souligner l'importance des notions d'identifiabilité et d'activation pour décrire la répartition des expressions référentielles dans les titres de presse. La prise en compte de ces deux catégories de la structure informationnelle (cf. Chafe, 1976 et Lambrecht, 1994) permet non seulement d'identifier deux types de titres dans lesquels l'expression référentielle initiale n'est pas soumise aux mêmes contraintes, les titres thétiques « ordinaires » et les titres thétiques à topique initial non lié, mais aussi, contrairement à une étude comme celle de Kronrod \& Engel (2001), fondée sur la théorie de l'accessibilité d'Ariel, de rendre compte des expressions référentielles spécialisées dans les premières mentions (les indéfinis à lecture existentielle). Après avoir soumis la théorie de l'accessibilité d'Ariel à l'épreuve des titres - à la manière de Kronrod \& Engel, mais avec des résultats, on le verra, très différents - on montrera donc que l'identifiabilité et l'activation référentielles sont deux catégories décisives pour expliquer le choix référentiel dans les titres de presse.

\section{Accessibilité référentielle}

Selon la théorie de l'accessibilité d'Ariel (1990), le choix de telle ou telle expression référentielle dépend du degré d'activation présumé du référent (ou, plus exactement, de sa représentation mentale) : si le locuteur présume que le référent est très actif dans la mémoire de son auditeur / lecteur, il choisira un marqueur de forte accessibilité pour le désigner (par exemple, un pronom personnel); si, au contraire, il estime son accessibilité mentale faible, il utilisera un marqueur de faible accessibilité (par exemple, un nom propre).

Quatre facteurs déterminent, chez Ariel, l'accessibilité d'un référent :

- la distance entre l'expression référentielle et sa dernière mention (son antécédent) : plus cette distance sera courte, plus le référent sera accessible ;

- la compétition entre plusieurs expressions référentielles susceptibles de constituer l'antécédent : plus il y aura d'antécédents possibles, moins le référent sera accessible ;

- la saillance du référent, c'est-à-dire sa topicalité, sa centralité dans le discours. La position syntaxique de sujet et le nombre de reprises référentielles sont des critères déterminants : plus un référent aura fait l'objet de mentions, de préférence en position de sujet, plus il sera accessible ;

- l'unité : un référent sera plus accessible si son antécédent fait partie de la même unité que son expression actuelle, c'est-à-dire, s'il fait partie du même « cadre / monde / point de vue / segment ou paragraphe » (Ariel 1990, 29). Il peut y avoir ici des recoupements avec le critère de la distance, pour lequel Ariel utilise déjà le paragraphe.

La relation entre les formes linguistiques et le degré d'accessibilité qu'elles codent n'est pas arbitraire. Elle répond à trois principes différents :

- un principe d'informativité, lié à la quantité d'information lexicale,

- un principe de rigidité, c'est-à-dire d'univocité, de non-ambiguïté de la forme, 
- un principe d'atténuation, c'est-à-dire de légèreté phonétique, en termes de longueur et d'accentuation.

Moins l'expression est informative, rigide et longue, plus son référent est présumé accessible. Inversement, plus une expression est informative, univoque et longue, moins son référent est présumé accessible. Les formes codant le plus haut degré d'accessibilité seront donc les moins informatives, les plus ambiguës et les plus courtes (formes zéros, pronoms atones...) et, inversement, les formes codant le plus faible degré d'accessibilité seront les plus informatives, les plus univoques et les plus longues.

Les expressions référentielles qu'on trouve dans les titres de presse marquent généralement un degré d'accessibilité moins faible que celui qu'on pourrait attendre en première mention et, le plus souvent, moins faible que l'expression référentielle qui inaugure l'article même. Le début d'article (1), titraille comprise, est représentatif de ce degré d'accessibilité décroissant :

(1) Philippe Courroye se dit victime d'un « lynchage » [titre]

Le procureur de Nanterre a demandé sa mise en disponibilité pour devenir avocat à Paris [sous-titre]

Philippe Courroye, le procureur de Nanterre, estime avoir été "lynché » et faire l'objet "d'une chasse à l'homme », dans un entretien publié mercredi 25 juillet par Le Figaro. [début de l'article] (Le Monde, 26 juillet 2012)

L'expression la plus explicite n'apparaît ni dans le titre ni dans le sous-titre, mais en troisième mention, au début de l'article même (cf. Jucker, 1996 ; Kronrod \& Engel, 2001 et Rebeyrolle et al., 2009, pour des observations similaires).

L'emploi des expressions référentielles dans les débuts d'articles de presse ne semble donc pas répondre aux prévisions de la théorie de l'accessibilité d'Ariel (1990, 1996 et 2008), qui ordonne de la manière suivante les expressions référentielles de (1):

(2)

$$
\begin{aligned}
& \text { description définie courte : Le procureur de Nanterre } \\
& \text { nom propre complet [prénom + nom de famille] : Philippe Courroye } \\
& \text { nom propre complet + modifieur : Philippe Courroye, le procureur de Nanterre }
\end{aligned}
$$

\section{Accessibilité minimale}

Dans l'exemple (1), la dernière expression référentielle (Philippe Courroye, le procureur de Nanterre) est la plus informative, la plus rigide et la plus longue, autrement dit elle code, selon les trois principes d'Ariel (informativité, rigidité et degré d'atténuation de la forme utilisée), le degré d'accessibilité référentielle le plus faible, après deux expressions référentielles qui, même si elles font partie, chez Ariel, des marqueurs de faible accessibilité, codent un degré d'accessibilité un peu moins faible.

On peut estimer que, avant leur deuxième mention, les référents ne sont pas encore cognitivement très accessibles et réclament ainsi d'être redonnés sous une forme codant un faible degré d'accessibilité (cf. Ariel, $1996: 33$, note 15$)^{1}$. Mais, après cette deuxième mention, l'utilisation, en troisième mention, de l'expression marquée du degré d'accessibilité le plus faible aurait de quoi surprendre dans tout autre contexte $^{2}$. Cette apparente violation de la théorie de l'accessibilité peut s'expliquer par le caractère périphérique de la titraille, c'est-à-dire son appartenance au paratexte, qui «n'est pas encore le texte » (Genette, 1987 : 13). L'expression référentielle la plus précise est en fait la première (et non la troisième) mention du texte à proprement parler. Cette différence essentielle de niveaux entre le texte et le paratexte peut aussi expliquer l'impossibilité, soulignée par Jucker (1996: 385 et 389), de commencer l'article - le texte donc - par un pronom anaphorique qui reprendrait une expression référentielle du titre. Mais cela n'explique pas pourquoi les expressions référentielles du titre ne marquent pas une accessibilité tout aussi faible. 
Selon Kronrod \& Engel (2001), l'utilisation dans les titres de marqueurs d'accessibilité moins faible serait le résultat d'un compromis entre deux tendances opposées : si l'accessibilité du référent appelle un marqueur de faible accessibilité, la volonté du rédacteur de ne pas prendre trop d'espace et d'éveiller la curiosité du lecteur en omettant certaines informations, appelle, elle, l'emploi de formes courtes, peu informatives et peu rigides, autrement dit de marqueurs de forte accessibilité. Le résultat du compromis entre d'une part l'accessibilité du référent et d'autre part la nécessité de brièveté et d'accroche des titres de presse serait donc d'employer des formes que Kronrod \& Engel (2001) qualifient d'intermédiaires : prénoms, noms de famille et descriptions définies courtes ${ }^{3}$ (on notera que les marqueurs d'accessibilité intermédiaire de Kronrod \& Engel restent des marqueurs de faible accessibilité chez Ariel).

Pour illustrer cette prédominance des emplois de marqueurs « intermédiaires », Kronrod \& Engel étudient les expressions référentielles dans les titres de trois quotidiens israéliens (en hébreu) - deux relevant de la presse populaire et un plus élitiste - parus le 24 janvier 1997. Elles n'observent pas de différences significatives entre ces trois quotidiens, ni entre les différentes rubriques (articles en une, sports, actualités, etc.) : à chaque fois, ce sont les marqueurs d'accessibilité «intermédiaire » qui prédominent (entre $69,2 \%$ et $75,2 \%$ selon les quotidiens), les marqueurs de forte accessibilité ${ }^{4}$ sont peu nombreux (5,6\% à 9,5\% des occurrences) sauf dans un des quotidiens populaires qui en compte $17,8 \%$, et les marqueurs de faible accessibilité - descriptions définies longues, noms propres complets et noms propres complets avec modifieur - représentent entre $13 \%$ et $19,2 \%$ des occurrences. L'expression référentielle la plus fréquente est la description définie courte (entre $28,1 \%$ et $35,5 \%$ ), suivie de près par le prénom ${ }^{5}$ (entre $24,5 \%$ et $33,2 \%$ ), et enfin le nom de famille (entre $12,3 \%$ et $15,7 \%$ ).

Observe-t-on la même tendance à privilégier les marqueurs d'accessibilité «intermédiaires » dans les titres de quotidiens français ? Un relevé des mêmes marqueurs d'accessibilité (moins les deux premiers marqueurs de forte accessibilité - formes zéro et accords en personne ; cf. le tableau 1 et les remarques qui suivent) dans les titres du Monde et de Libération du 26 juillet 2012 ne conduit pas du tout aux mêmes types de résultats. 
Tableau 1 - Marqueurs d'accessibilité retenus

Marqueurs d'accessibilité (par ordre d'accessibilité décroissante)

Pronom personnel

Prénom

Nom de famille

Description définie courte (jusqu'à 2 unités lexicales)

Description définie longue (3 unités lexicales et plus)

Nom propre complet (prénom+nom de famille, pseudonyme, toponyme, nom d'entreprise ou d'organisation...)

Nom propre complet + modifieur
Exemples (Le Monde et Libération)

$I l ; j e$

Nicolas

Hollande

Le décret; le gouvernement; les voitures propres; la panoplie de l'été

L'arsenal chimique syrien; la réforme de la politique agricole commune; le débat sur les gaz de schiste

Ségolène Royal ; Madonna ; Alep ; l'Iran ; EDF ; Air France

Rodrigo Rata, l'ancien patron de Bankia ; La coopérative Sofiprotéal

Remarques sur certaines des expressions référentielles relevées :

- Pronom personnel : il s'agit soit de pronoms anaphoriques trouvant leur antécédent dans le titre (ex. PSA [groupe automobile] estime qu'il doit réaliser 1,5 milliard d'euros d'économies d'ici à 2005; Le Monde), soit de pronoms déictiques dans une citation (ex. En France, je n'aurais pas osé; Le Monde).

- Prénom : 1 seule occurrence et encore celle-ci est très particulière : elle est liée à un jeu de mots qui laisse apparaître le nom de famille, Tourner comme Nicolas en Cage (Libération).

- Nom propre complet + modifieur : dans cette catégorie, on trouve à la fois des noms propres complets accompagnés d'une apposition et des noms propres complets accompagnés d'un nom qui précise une classe d'appartenance ; en termes d'informativité, de rigidité et de longueur on peut rapprocher les deux constructions (c'est-à-dire rapprocher l'acteur américain Nicolas Cage et Nicolas Cage, l'acteur américain).

- Le relevé exclut quelques expressions référentielles, certaines rares (un pronom interrogatif dans $L e$ Monde, un SN démonstratif dans Libération), d'autres fréquentes (des SN indéfinis ou numéraux et des SN construits sans déterminant, se rapprochant soit de descriptions définies - ex. Mort du président Atta Mills; Le Monde - soit de descriptions indéfinies - ex. Arrestations aux obsèques du dissident cubain Oswaldo Paya ; Le Monde). 
- Les expressions référentielles enchâssées n'ont pas été comptées séparément : par exemple, pour la riposte du régime de Damas (Le Monde), une seule expression référentielle, description définie longue, a été comptée et non pas trois, qui seraient la riposte du régime de Damas, le régime de Damas et Damas. Suivant le même principe, les déterminants possessifs n'ont pas été relevés séparément de l'expression référentielle dans laquelle ils apparaissent. Par exemple, dans le SN ses centrales nucléaires (Le Monde), une seule expression référentielle, description définie courte avec deux unités lexicales, a été prise en compte. C'est-à-dire que la part pronominale du déterminant possessif («d'elle » dans ses centrales nucléaires) n’a pas été pas comptée séparément, de la même manière que si l'on avait eu affaire à un $\mathrm{SN}$ comme les centrales nucléaires d'EDF, on n'aurait pas compté séparément le nom propre $E D F{ }^{6}$ Les déterminants possessifs se révèlent peu nombreux (2 occurrences dans les titres du Monde et 3 dans ceux de Libération) et, à une exception près (un emploi générique dans l'expression idiomatique qui constitue le titre Abattre son joker de Libération), ils trouvent tous leur antécédent dans le titre même (ex. EDF néglige le droit du travail dans ses centrales nucléaires; Le Monde).

Les résultats obtenus sont, comme chez Kronrod \& Engel, donnés en deux tableaux. Le premier tableau (tableau 2) présente tous les marqueurs d'accessibilité pris en considération et le second (tableau 3) les rassemble en trois catégories: marqueurs de forte accessibilité (pronoms personnels), marqueurs d'accessibilité intermédiaire (prénom, nom de famille, description définie courte) et marqueurs de faible accessibilité (description définie longue, nom propre complet et nom propre complet + modifieur). Les pourcentages sont arrondis. 
Tableau 2 - Résultats pour tous les marqueurs d'accessibilité retenus

$\begin{array}{llllllll}\text { Total des } & \text { Pronom } & \text { Prénom } & \begin{array}{l}\text { Nom de } \\ \text { famille }\end{array} & \begin{array}{l}\text { Description } \\ \text { définie }\end{array} & \begin{array}{l}\text { Description } \\ \text { définie }\end{array} & \begin{array}{l}\text { Nom } \\ \text { propre }\end{array} & \begin{array}{l}\text { Nom } \\ \text { propre } \\ \text { complet }+ \\ \text { d'accessibilité }\end{array} \\ \text { personnel } & & & \text { courte } & \text { longue } & \text { complet } & \begin{array}{l}\text { complifieur } \\ \text { mode }\end{array}\end{array}$

$\begin{array}{llllllll}\text { Le Monde } & 3,8 \% & 0 & 2,8 \% & 39 \% & 18 \% & 28,6 \% & 7,6 \% \\ (105) & (4) & & (3) & (41) & (19) & (30) & (8)\end{array}$

$\begin{array}{llllllll}\text { Libération } & 1,9 \% & 1,9 \% & 15 \% & 34 \% & 9,4 \% & 30,2 \% & 7,5 \% \\ (53) & (1) & (1) & (8) & (18) & (5) & (16) & \text { (4) }\end{array}$

Tableau 3 - Résultats pour ces mêmes marqueurs d'accessibilité rassemblés en trois catégories

Marqueurs de forte accessibilité
Marqueurs d'accessibilité intermédiaire
Marqueurs de faible accessibilité
Le Monde $\quad 3,8 \%$

(105)

(4)

$1,9 \%$

(1)

(53)
$41,9 \%$

(44)

$50,9 \%$

$47,2 \%$
$54,2 \%$

(57)

Ces résultats sont très différents de ceux de Kronrod \& Engel. Ils sont aussi assez différents de ceux de Jucker (1996), qui, lui, relève une forte proportion de noms de famille dans les titres (relevés effectués dans sept quotidiens britanniques), mais qui ne s'attache qu'aux expressions référant à des humains ${ }^{7}$.

Dans Le Monde et Libération, l'emploi des marqueurs d'accessibilité intermédiaire n'est pas dominant. En fait, les pourcentages entre marqueurs d'accessibilité intermédiaire et marqueurs de faible accessibilité sont assez proches (surtout dans Libération), mais ceux de faible accessibilité seraient bien plus importants si l'on n'avait pas ignoré certaines expressions référentielles, comme les SN indéfinis et numéraux ou certains SN construits sans déterminant. L'expression référentielle la plus fréquente est aussi la description définie courte (entre $34 \%$ et $39 \%$ ), suivie par le nom propre complet (entre $28,6 \%$ et $30,2 \%)$. 
Par ailleurs, classer les descriptions définies courtes parmi les marqueurs d'accessibilité intermédiaire est souvent contestable, surtout du point de vue de la rigidité (le deuxième principe d'Ariel, lié à l'univocité de la forme). En fait, la plupart des descriptions définies courtes relevées dans les titres du Monde et de Libération sont des descriptions définies complètes (ex. les voitures propres, avec une interprétation générique, dans L'Etat parie sur les voitures propres; Le Monde), ou semi-complètes ${ }^{8}$ (ex. le gouvernement dans Le gouvernement fait le pari de l'automobile verte; Le Monde), plus courtes que les descriptions définies longues, mais tout aussi rigides. Très peu sont des descriptions incomplètes (ex. Les taux épargnent le Nord, étrillent le Sud; Libération; ici c'est l'article qui permettra de donner une interprétation aux SN du titre : les taux d'intérêt des dettes souveraines, le Nord et le Sud de l'Europe) et, très peu finalement marquent véritablement un degré d'accessibilité intermédiaire.

La presse à scandale opère, semble-t-il, des choix référentiels très différents. Les titres relevés dans France-Dimanche, Ici-Paris, Allo police et Qui ?, par Charaudeau (1983) et repris par Sullet-Nylander (1998: 40), présentent un grand nombre de marqueurs de forte accessibilité et d'accessibilité intermédiaire, pronoms de $3^{\text {ème }}$ personne sans antécédent et «vraies » descriptions définies courtes, c'està-dire incomplètes : Elle paie un tueur pour se faire assassiner, Il exécute son rival en l'opérant, Le satyre avait peur des femmes, La machine le prive à vie de l'usage de ses mains. Autant de pseudoanaphores, ce qu'on pourrait appeler des anaphores in medias res, qui plongent le lecteur dans le vif du sujet.

Comparés aux résultats de Kronrod \& Engel, nos résultats confortent donc davantage la thèse d'Ariel, en montrant que l'accessibilité du référent reste le facteur le plus important pour le choix référentiel dans les titres, du moins dans ceux de quotidiens comme Le Monde et Libération. Autrement dit, ces résultats n'appuient pas la thèse du compromis censé résoudre le conflit entre d'une part l'accessibilité du référent et d'autre part la nécessité de brièveté et d'accroche des titres de presse. Dans Le Monde et Libération, ce n'est que la décroissance du niveau d'accessibilité entre la titraille et le début de l'article qui semble peu conforme aux prévisions de la théorie d'Ariel, mais, comme on l'a déjà souligné, l'emploi d'un marqueur de (très) faible accessibilité référentielle au début de l'article, après une ou deux mentions dans la titraille, peut s'expliquer par le caractère périphérique de cette dernière. La décroissance du niveau d'accessibilité, quant à elle, est liée aux conventions de spécification croissante (i.e. aux conventions élaborantes; voir Schnedecker, 2005 ; Salles, 2011) qui caractérisent les différents niveaux de lecture des débuts d'articles de presse

\section{Identifiabilité et activation référentielles}

La théorie de l'accessibilité ne permet toutefois pas de rendre compte de tous les choix référentiels. Selon Chafe (1994 : 179), «bien qu'Ariel mentionne différents degrés de « disponibilité mémorielle » », ce qui est réellement en jeu dans sa théorie, c'est «la nature du langage nécessaire pour rendre un référent partagé identifiable dans un contexte donné ». Son échelle, qui va des expressions qui réclament le plus d'information verbalisée pour la bonne identification d'un référent partagé à celles qui réclament peu (voire pas, avec les formes zéro) d'information verbalisée, ordonne ainsi des expressions aux référents identifiables (Ariel $1990: 73)$ :

\section{Accessibilité minimale}

Nom propre complet + modifieur

Nom propre complet

Description définie longue

Description définie courte

Nom patronymique

Prénom 


\author{
Démonstratif d'éloignement + modifieur \\ Démonstratif de proximité + modifieur \\ Démonstratif d'éloignement \\ Démonstratif de proximité \\ Pronom accentué avec geste \\ Pronom accentué \\ Pronom atone \\ Pronom clitique
}

Marqueurs d'accessibilité très élevée (éléments vides, comprenant pro, PRO...)

\title{
Accessibilité maximale
}

Les expressions indéfinies, dont le référent n'est pas toujours identifiable (il ne l'est pas lorsque celles-ci reçoivent une interprétation spécifique existentielle), n'ont pas leur place dans cette échelle, alors qu'elles constituent une part relativement importante des expressions qu'on peut relever dans les titres de presse. Il existe, en outre, sur l'emploi de ce type d'expressions référentielles, des contraintes que la théorie de l'accessibilité est incapable d'expliquer. La prise en compte de deux catégories de la structure informationnelle, l'identifiabilité du référent et son activation (Chafe, 1976, 1994), permet, au contraire, de bien distinguer les facteurs qui déterminent le choix référentiel dans les titres de presse. Ces deux catégories sont définies ainsi par Lambrecht (1994: 76) :

- l'identifiabilité concerne l'évaluation par un locuteur de la présence ou non dans l'esprit de l'auditeur d'une représentation de discours du référent. Un référent identifiable est un référent pour lequel il existe déjà une représentation partagée par le locuteur et l'auditeur au moment de l'acte de parole, alors qu'un référent non identifiable est un référent dont seul le locuteur a une représentation ;

- l'activation concerne l'évaluation par un locuteur du statut de la représentation d'un référent identifiable comme déjà activée, simplement accessible ou inactive dans l'esprit de l'auditeur au moment de l'acte de parole. La représentation d'un référent est active lorsqu'elle est actuellement «allumée » dans l'esprit de l'auditeur, pour reprendre la métaphore de Chafe. Elle est accessible (ou semi-active) lorsqu'elle n'est pas directement dans le focus d'attention, mais en arrière-plan, dans une zone périphérique de la conscience, et inactive lorsqu'elle est disponible dans la mémoire à long terme seulement (cf. la présentation des trois statuts d'activité de Chafe par Lambrecht, 1994 : 94).

La forme des éléments initiaux des titres de presse est, comme on va le voir, conditionnée par deux caractéristiques ayant des incidences l'une sur l'identifiabilité du référent, l'autre sur son activation :

- leur statut généralement non topical permet l'utilisation d'expressions aux référents non identifiables pour le lecteur (des expressions «brand-new unanchored» chez Prince, 1981), sans représentation déjà disponible dans l'esprit du lecteur ;

- le fait qu'elles constituent généralement la toute première mention d'un référent a pour corollaire le fait que, si le référent en question est identifiable, alors il est inactif (c'est-à-dire que sa représentation est inactive dans l'esprit du lecteur au moment de l'occurrence de son expression). Sauf cas de reprise à l'intérieur même du titre (ex. PSA estime qu'il doit réaliser 1,5 milliard d'euros d'économie d'ici à 2005 ; Le Monde), on ne trouvera donc guère d'expressions codant le statut actif comme les pronoms personnels dans les titres de presse (presse à scandale exceptée). 


\subsection{Statut non topical des expressions référentielles dans les titres de presse}

Les titres de presse sont des énoncés thétiques, c'est-à-dire des énoncés dont la « raison d'être au niveau discursivo-pragmatique [...] est de servir à présenter une entité, une proposition ou un état de choses en tant qu'élément d'information nouveau pour le discours. » (Cornish, 2008 : 122).

Dans l'interprétation «titre de journal» envisagée par Gundel \& Fretheim (1993) de l'exemple de Lambrecht (1994), Nazis tear down antiwar posters, on a affaire à une phrase thétique (du type « eventreporting »; cf. Lambrecht, 1994), la phrase entière est le focus. Le topique, ce dont on parle et ce dont on accroît la connaissance ${ }^{9}$, est simplement ce qui est arrivé aujourd'hui. Lorsque les titres présentent une structure sujet-prédicat, le sujet fait donc partie du focus et n'est pas le topique ${ }^{10}$.

Dans la mesure où il n'est pas le topique, le sujet n'est soumis à aucune contrainte référentielle : son référent n'exige pas d'être identifiable, c'est-à-dire que le locuteur n'a pas besoin de présumer une représentation disponible dans l'esprit de l'auditeur / lecteur. En d'autres termes, il échappe à la seule véritable contrainte référentielle, l'identifiabilité, sur les types de SN pouvant remplir la position de topique. $^{11}$

On peut alors aussi bien trouver des SN indéfinis spécifiques à lecture existentielle (exemples (3)), dont les référents ne sont pas encore identifiables pour le lecteur (ce dernier ne dispose pas alors d'une représentation de ces référents) que des SN définis ou des noms propres (exemples (4)) aux référents identifiables, en position de sujet ${ }^{12}$ :

(3) a. Une nouvelle crèche clandestine découverte à Marseille (Le Monde, 26 juillet 2012)

b. Des archéologues italiens sur les traces de « la Joconde » à Florence (Le Monde, 26 juillet 2012)

(4) a. Le gouvernement fait le pari de l'automobile verte (Le Monde, 26 juillet 2012)

b. La zone euro presse la BCE de voler au secours de l'Espagne (Le Monde, 26 juillet 2012)

c. Alep se prépare à la riposte du régime de Damas (Le Monde, 26 juillet 2012)

Les $\mathrm{SN}$ des titres de presse, quelle que soit leur position syntaxique, ne posent-ils pour autant aucune contrainte quant à l'identifiabilité de leurs référents?

\subsection{Un autre type de titre : des énoncés thétiques à topique initial}

On observe une structure récurrente dans les titres de presse qui semble exiger une identifiabilité du référent initial. Cette structure, dite «bisegmentale » par Bosredon \& Tamba (1992) ${ }^{13}$, présente un SN initial suivi de deux points introduisant soit une structure phrastique complète ou à ellipse du verbe être (5), soit un SN ou des SN coordonnés $(6)^{14}$ :

(5) a. Doux : la coopérative Soliprotéal et Charles Doux vont s'affronter pour la reprise du travail (Le Monde, 26 juillet 2012)

b. Salaires : Bailly suit le décret à la lettre (Le Monde, 26 juillet 2012)

c. Dépassements d'honoraires : les médecins sur la défensive (Le Monde, 26 juillet 2012)

d. Affaire Halliday : l'avocat répond au procureur (Le Monde, 20-21 février 2005)

(6) a. Nucléaire: les infractions d'EDF au droit du travail (Le Monde, 26 juillet 2012)

b. Cour de justice de la République : sept PS, quatre UMP et un centriste (Le Monde, 26 juillet 2012)

Les énoncés sont bien thétiques dans la mesure où leur objet est de présenter « une proposition ou un état de choses en tant qu'élément d'information nouveau pour le discours. " (cf. Cornish $2008: 122$, déjà cité). Conformément à la caractérisation des énoncés thétiques faite par Lambrecht (1994), s'il y a un sujet grammatical (exemples (5)), celui-ci n'est pas un topique, mais fait partie du contenu asserté, non présupposé, c'est-à-dire du focus. Il n'est donc pas soumis à la contrainte d'identifiabilité et pourrait être 
réalisé, même si ce n'est pas le cas dans les exemples relevés, par des SN indéfinis ou numéraux à lecture existentielle; par exemple :

(7) a. Affaire Halliday : un témoin devrait être entendu demain par le procureur

b. Cour de justice de la République : sept PS, quatre UMP et un centriste jugeront leurs pairs

En revanche, de tels SN semblent exclus de la position initiale de ces énoncés. Tous les SN initiaux de ces titres ont des référents identifiables. Seule cette contrainte d'identifiabilité pèse sur le type de SN pouvant être topique, ce qui n'exclut pas les SN sans déterminant de (5b-d)-(6) (auxquels Bosredon \& Tamba 1992: 37, refuseraient pourtant le statut de thème, en partie à cause de cette forme non déterminée). Deux types de SN sont utilisés : des noms propres ou, le plus souvent, des SN construits sans déterminant. Aucun de ces SN sans déterminant n'a une référence indéfinie.

Il n'est pas impossible d'imaginer des titres apparemment proches des précédents avec un SN indéfini ou numéral initial :

(8) a. Un rebondissement dans l'affaire Halliday : un nouveau témoin devrait être entendu demain par le procureur

b. Douze élus à la Cour de justice de la République : sept PS, quatre UMP et un centriste

Mais la structure informationnelle de ces titres-là se révèle très différente de celle des précédents : les éléments initiaux de (8) constituent clairement le focus, le contenu asserté, la suite (après les deux points) ne faisant que préciser quelle est la nature du rebondissement, quels sont les douze élus. Ces éléments initiaux pourraient constituer le titre à eux seuls. Dans les titres présentés en (5)-(6), en revanche, ce qui suit l'élément initial ne constitue pas une spécification de ce qui précède et l'élément initial ne saurait constituer à lui seul le titre. Il ne pourrait à lui seul recevoir une interprétation thétique et ne saurait répondre à une question comme Qu'est-il arrivé ?/Que s'est-il passé ?/Quoi de neuf?

(9) Qu'est-il arrivé ?/Quoi de neuf ?

a. (Il y a) Un rebondissement dans l'affaire Halliday

b. \# Affaire Halliday

En fait, si l'on devait utiliser le test question-réponse pour identifier le focus des énoncés présentés en (5)(6), une question comprenant l'élément initial conviendrait davantage, la réponse se limitant à la suite du titre :

(10) Quoi de neuf à propos de l'affaire Halliday/de Doux ?

a. (A propos de l'affaire Halliday,) l'avocat répond au procureur

b. (A propos de Doux,) la coopérative Soliprotéal et Charles Doux vont s'affronter pour la reprise du travail

L'élément initial constitue une forme de rubrique ${ }^{15}$ pour ce qui suit et c'est aussi une entité, un événement ou un état de choses dont le lecteur a déjà une représentation (un sujet de société ou un sujet qui fait l'actualité du moment), autrement dit identifiable. Le test question-réponse souligne le statut topical de ces éléments initiaux : ce dont on parle, c'est de l'affaire Halliday, de l'entreprise Doux et ce qui suit est bien «A PROPOS de ce référent», exprime bien « une information qui est PERTINENTE POUR ce référent et qui accroît la CONNAISSANCE qu'a l'interlocuteur de ce référent", conformément à la définition donnée par Lambrecht (1994 : 126).

Si la paraphrase en à propos de proposée en (10a-b) peut être considérée comme une mise en relief du topique, alors ces SN initiaux sont bien des formes de topiques. Cette paraphrase rappelle les tests d'identification du topique en as for (Kuno, 1972 ; Gundel, 1974, Reinhart, 1981 ; Gundel \& Fretheim, 1993 ; Lambrecht, 1994) et en about (Reinhart, 1981 ; Lambrecht, 1994). Mais, contrairement à ces tests (11a-b), l'élément introduit par à propos de n'est pas repris dans la suite de l'énoncé : 
(11) a. As for Rosa, John didn't really love her.

b. John said about Rosa that he didn't really love her.

(Lambrecht 1994, 149)

Un détachement initial en quant à en français semble aussi exiger une reprise (la construction serait alors forcément une forme de construction disloquée) :

(12) a. Quant à Rosa, John ne l'aime pas vraiment.

b. ? Quant à l'affaire Halliday, l'avocat répond au procureur

c. ? Quant à Doux, la coopérative Soliprotéal et Charles Doux vont s'affronter pour la reprise du travail

En outre, alors que à propos de semble pouvoir être un simple introducteur de topique, le détachement en quant $\grave{a}$ indique un changement de topique parmi un ensemble de topiques déjà disponibles, ce qui suppose un contexte discursif antérieur et explique que l'expression référentielle introduite par quant à, comme celle du as-for test, doive avoir un référent non seulement identifiable (comme tout topique), mais aussi un référent qui ne soit pas inactif. Comme le rappelle Lambrecht (1994 : 152), qui s'appuie ici sur les travaux d'Ochs Keenan \& Schieffelin (1976), «le syntagme As for SN (tout comme des syntagmes similaires dans d'autres langues) ne peut être utilisé de façon appropriée que si le référent du SN est déjà un topique potentiel du discours au moment où ce syntagme est utilisé, autrement dit lorsque ce référent est contextuellement accessible ». Ainsi l'énoncé As for your brother, I saw him last night, ajoute Lambrecht, «n'est approprié que si le frère appartient à l'ensemble des référents dont on discute ». On peut dire la même chose d'un énoncé comme Quant à ton frère, je l'ai vu hier soir.

Nos paraphrases en à propos de ne posent pas ce type de contraintes : dans la mesure où elles n'indiquent pas un changement de topique parmi un ensemble de topiques déjà disponibles et ne supposent pas ainsi un discours préalable, elles n'exigent pas un statut d'activation particulier du référent. Ce dernier a seulement besoin, en tant que topique, d'être identifiable, mais il peut très bien être inactif. Il l'est dans la plupart de nos titres ${ }^{16}$.

Ces topiques, à l'initiale d'un énoncé thétique, répondent bien à la définition de topique que donne Chafe (1976), en termes d'expression « scene setting », c'est-à-dire d'expression qui installe « un cadre spatial, temporel ou individuel dans lequel a lieu la prédication principale » et qu'il distingue, comme le rappelle Lambrecht (1994: 118), du «sujet», caractérisé comme «point d'ancrage pour la nouvelle connaissance » (Chafe, 1976, cité par Lambrecht). Selon Lambrecht, c'est une façon pour Chafe de distinguer les topiques qui ne sont pas intégrés syntaxiquement et sémantiquement dans la structure prédicat-argument(s) d'une proposition de ceux qui le sont. La notion de topique qu'utilise Lambrecht recouvre, quant à elle, ces deux types de topiques (arguments ou non). Les référents des expressions nominales initiales des exemples (5)-(6) sont interprétés comme des topiques dans la mesure où ce qui suit est bien à propos d'eux.

Ce type de titre peut être rapproché des «constructions à topique non lié » (« unlinked topic constructions ») de Lambrecht (1994 : 193), constructions, fréquentes à l'oral, dans lesquelles un élément nominal (Dade County, The typical family today, Tulips) est détaché sans être repris par un élément pronominal dans la proposition qui suit :

(13) a. Dade County, you just can’t believe the rise in crime.

b. That isn't the typical family anymore. The typical family today, the husband and the wife both work.

c. Tulips, you have to plant new bulbs every year?

(exemples relevés par Lambrecht 1994 : 193)

L'absence de reprise pronominale de ces SN initiaux exclut toute position argumentale des topiques auxquels ils réfèrent dans la proposition. Les titres (5)-(6) seraient ainsi des formes de constructions à topique non lié, propres cette fois non à l'oral, mais aux titres journalistiques. Ils paraissent très différents 
des titres d'articles de linguistique qui utilisent les deux points, même de ceux qui utilisent une préposition « topicale » comme sur :

(14) a. Sur l'anaphore associative : article défini et adjectif démonstratif

b. Sur le sens du sens : contre la représentation sémantique des noms chez Putnam.

(titres de deux articles de Kleiber)

Dans ces exemples, sur n'introduit pas le premier membre comme topique par rapport au second, qui serait le focus. Les deux membres définissent ensemble le topique global de l'article et, contrairement aux titres à topique initial non lié, le premier membre de (14a-b) pourrait constituer à lui seul le titre de l'article.

\section{Conclusion}

Les relevés des expressions référentielles dans les titres du Monde et de Libération confortent assez bien la thèse d'Ariel : on trouve majoritairement des marqueurs de faible accessibilité, surtout lorsqu'on élargit cette catégorie à d'autres marqueurs, comme la plupart des descriptions définies courtes. Kronrod \& Engel (2001) traitent uniformément les descriptions définies courtes (jusqu'à deux unités lexicales) comme des marqueurs d'accessibilité intermédiaire, alors que la plupart de celles qu'on peut relever dans les titres de presse sont, malgré une forme plus courte, tout aussi rigides et univoques que des descriptions définies plus longues.

Le même relevé dans d'autres types de presse conduirait probablement à des résultats très différents : la presse à scandale multiplie, on l'a constaté avec les titres relevés par Charaudeau (1983), l'emploi de pseudo-anaphores, marqueurs d'accessibilité intermédiaire ( « vraies 》 descriptions définies courtes cette fois, c'est-à-dire incomplètes: Le satyre avait peur des femmes) et marqueurs de forte accessibilité (pronoms personnels: Il exécute son rival en l'opérant). L'utilisation transgressive d'un marqueur d'accessibilité forte ou intermédiaire pour un référent qui n'est pas encore accessible produit un effet d'accroche bien plus spectaculaire que celui évoqué par Kronrod \& Engel (2001), un effet qu'on pourrait décrire à la manière de Tom Wolfe : «Pensez-donc à ce superbe sens de la syntaxe du caniveau qui les avait inspirés pour créer un titre fait de verbes et d'objets, où le vrai sujet manquait, meilleur moyen de vous faire plonger vos griffes dans ces pages noires et grasses pour savoir quel démon était assez monstrueux pour être ce «IL »» (Le Bûcher des vanités, traduction française B. Legrand, Le Livre de poche, 229-230), le IL du titre IL SCALPE GRAND-MERE ET LA DEPOUILLE.

La théorie de l'accessibilité référentielle ne permet cependant pas de rendre compte de tous les choix référentiels possibles : il n'y a pas de place dans l'échelle d'accessibilité pour les formes spécialisées dans les mentions initiales (les indéfinis existentiels), qui constituent une part non négligeable des expressions référentielles relevées dans les titres de presse et qui sont par ailleurs exclues de la position initiale de certains titres. La prise en compte des deux catégories de Chafe (1976), l'identifiabilité et l'activation référentielles, permet, au contraire, de décrire l'ensemble des choix référentiels possibles et de souligner des différences de contraintes selon la strucutre informationnelle des titres - thétiques ordinaires ou thétiques à topique initial non lié.

Les titres thétiques ordinaires n'imposent aucune restriction sur l'identifiabilité des référents et privilégient l'emploi d'expressions dont les référents sont inactifs. Hormis quelques cas de reprises à l'intérieur même d'un titre (ex. Mort du président Mills, le vice-président lui succède, Philippe Courroye se dit victime d'un "lynchage », Le Monde, 26 juillet 2012), rares seront les formes habituellement associées aux référents actifs comme les pronoms personnels.

Les titres à topique initial non lié imposent, eux, une contrainte d'identifiabilité sur le premier référent. Celui-ci doit, en effet, être identifiable, contrainte généralement admise pour les topiques. Et, dans la plupart des cas, il sera lui aussi inactif. Cette contrainte d'identifiabilité exclut de la position initiale les SN indéfinis qu'on peut trouver à l'initiale des titres thétiques ordinaires (ex. Des archéologues italiens sur les traces de «la Joconde» à Florence, Le Monde, 26 juillet 2012). Une construction avec un SN 
indéfini initial comme Des archéologues italiens : sur les traces de «la Joconde » à Florence ne semble pas acceptable. Et un titre comme Archéologues italiens : sur les traces de «la Joconde » à Florence supposerait une identifiabilité du référent, une référence définie introduisant un nouvel épisode du feuilleton des archéologues italiens et de leur quête du modèle de la Joconde.

\section{Références bibliographiques}

Ariel, M. (1990). Accessing Noun-Phrase Antecedents. London/New York : Routledge

Ariel, M. (1996). Referring Expressions and the +/- Coreference Distinction. Reference and Referent Accessibility, T. Fretheim \& J.K. Gundel (éds). Amsterdam : John Benjamins, 13-33.

Ariel, M. (2008). Pragmatics and Grammar. Cambridge: Cambridge University Press.

Bosredon, B. \& Tamba, I. (1992). Thème et titre de presse : les formules bisegmentales articulées par un « deux points ». L'information grammaticale, 54, 36-44.

Chafe, W. (1976). Givenness, contrastiveness, definiteness, subjects, topics, and point of view. Subject and topic, C.N. Li (éd.). New York : Academic Press, 25-56.

Chafe, W. (1994). Discourse, consciousness, and time. Chicago : The University of Chicago Press.

Charaudeau, P. (1983). Langage et discours. Eléments de sémiolinguistique. Paris : Hachette.

Cornish, F. (2008). L'absence de prédication, le topique et le focus : le cas des phrases thétiques. Faits de langue, 31-32, 121-131.

Genette, G. (1987). Seuils. Paris : Points/Editions du Seuil.

Gundel, J. (1974). The role of topic and comment in linguistic theory. Ph. D. Dissertation, University of Texas at Austin.

Gundel, J. (1985). Shared knowledge and topicality. Journal of Pragmatics, 9, 83-107.

Gundel, J. \& Fretheim, T. (1993). Topic and focus. Handbook of pragmatics, G. Ward \& L. Horn (eds). Londres : Blackwell, 175-196.

Jucker, A. H. (1996). News actor labelling in British newspapers. Text, 16.3, 373-390.

Kleiber, G. (1981). Problèmes de référence : descriptions définies et noms propres. Paris : Klincksieck.

Kronrod, A. \& Engel, O. (2001). Accessibility theory and referring expressions in newspaper headlines. Journal of Pragmatics, 33, 683-699.

Kuno, S. (1972). Functional sentence perspective : a case study from Japanese and English. Linguistic Inquiry, 3, 269-320.

Lambrecht, K. (1994). Information structure and sentence form. Cambridge : Cambridge University Press.

Ochs Keenan, E. \& Schieffelin, B. (1976). Topic as a discourse notion: a study of topic in the conversations of children and adults. Subject and topic, C.N. Li (eéd.). New York : Academic Press, 335384.

Prince, E. (1981). Toward a taxonomy of given-new information. Radical Pragmatics, P. Cole (ed.). Academic Press : New York, 223-255.

Rebeyrolle, J., Jacques, M.-P. \& Péry-Woodley, M.-P. (2009). Titres et intertitres dans l'organisation du discours. Journal of French Language Studies, 19.2, 269-290.

Reinhart, T. (1981). Pragmatics and linguistics : an analysis of sentence topics. Philosophica, 27, 1, 5394. 
Salles, M. (2011). Que présuppose l'anaphore dite présuppositionnelle ? Sur la coréférenciation des expressions nominales complètes. Journal of French Language Studies, 21.2, 191-208.

Schnedecker, C. (2005). Les chaînes de référence dans les portraits journalistiques : éléments de description. Travaux de linguistique, 51, 85-133

Sullet-Nylander, F. (1998). Le titre de presse. Analyses syntaxique, pragmatique et rhétorique. Stockholm : Institutionen för franska och itlienska, Stockholm Universitet.

\begin{abstract}
${ }^{1}$ Ariel $(1996,33)$ note le «besoin de répéter la référence aux nouvelles entités de discours par des SN lexicaux (marqueurs de faible accessibilité) plus d'une fois avant qu'elles ne soient considérées comme hautement accessibles ». C'est le troisième facteur, celui de la saillance, qui paraît essentiel ici : puisque la saillance est en partie liée aux nombre de reprises référentielles, un référent qui n'a encore été mentionné qu'une fois manque de saillance.

${ }^{2}$ Ce qui répond, en revanche, à la tendance générale, c'est l'emploi de SN pleins (à la place de pronoms) à chaque nouvelle unité textuelle, en l'occurrence à chaque «niveau de lecture » (titre, sous-titre, article), conformément au facteur unité de la théorie de l'accessibilité (facteur selon lequel le référent est plus accessible lorsqu'il a déjà été mentionné dans la même unité, c'est-à-dire dans le même cadre, monde, point de vue, segment ou paragraphe).
\end{abstract}

${ }^{3}$ Les descriptions définies courtes de Kronrod \& Engel sont des descriptions définies comprenant deux unités lexicales au maximum ; les descriptions définies longues sont celles qui comprennent trois unités lexicales et plus. La distinction est similaire à celle d'Ariel (1990).

${ }^{4}$ Il s'agit, dans les relevés de Kronrod \& Engel, de formes zéro, d'accord en personne (lorsqu'un nom ou un verbe contient un accord en personne ; exemple $t i k+o=$ sac + son », « son sac ») et de pronoms personnels.

${ }^{5}$ Cette forte proportion de prénoms est assez surprenante : en fait, la plupart des prénoms (ou surnoms) relevés par Kronrod \& Engel sont utilisés pour référer au même individu, le premier ministre de l'époque. Selon Kronrod \& Engel (2001: 693) cet emploi fréquent du prénom et encore plus du surnom (Bibi) pour désigner ce premier ministre est probablement lié à son jeune âge.

${ }^{6}$ De ce point de vue, la méthode de comptage adoptée ici semble différente de celle de Kronrod \& Engel (qui n'est pas très explicite), puisque, on l'a vu, leur relevé inclut des formes d'accord en personne associées à un nom (cf. note 4 et l'exemple $t i k+o=\langle\operatorname{sac}+\operatorname{son} »$, « son sac »).

${ }^{7}$ L'étude de Jucker (1996), tout comme celle de Schnedecker (2005), est consacrée à la répartition des expressions référentielles dans les titres et articles de presse (analyse des expressions référant à des humains dans les titres et articles de sept quotidiens britanniques par Jucker et dans les portraits journalistiques du Monde par Schnedecker) et non limitée comme la nôtre aux seuls titres.

${ }^{8}$ La complétude des descriptions définies «complètes » connaît des degrés, selon la capacité qu'a l'expression référentielle d'identifier seule son référent, indépendamment de tout ancrage contextuel ou non : cf. la distinction chez Kleiber (1981 : 250 et suivantes) entre l'ancrage per se que manifeste une description définie comme l'auteur des Misérables et l'ancrage contextuel que réclament des descriptions définies comme le président des Etats-Unis ou le gouvernement. La première est totalement autonome, les secondes restent dépendantes de leur contexte d'énonciation (les référents visés ne peuvent être identifiés sans une prise en compte de leur contexte d'énonciation, qui leur fournit l'ancrage - temporel pour le président des Etats-Unis, temporel et locatif pour le gouvernement nécessaire). La première est donc plus rigide au sens d'Ariel que les secondes, et, comme on le constate, ce n'est pas forcément une affaire de longueur.

${ }^{9}$ Cf. à ce égard la définition de Lambrecht (1994: 126) : «Un référent est interprété comme le topique d'une proposition si DANS UN DISCOURS DONNE la proposition est interprétée comme étant A PROPOS DE ce référent, i.e. comme exprimant une information qui est PERTINENTE POUR ce référent et qui accroît la CONNAISSANCE qu'a l'interlocuteur de ce référent. »

${ }^{10}$ On ne suit pas ici l'analyse de Sullet-Nylander (1998), qui identifie une structure thème/rhème dans quasiment tous les types de titres de presse, le thème et le rhème du titre s'identifiant finalement avec le thème global et le rhème global de l'article. 


\footnotetext{
${ }^{11}$ Selon Gundel (1985), en effet, l'expression référant au topique doit être définie ou générique, c'est-à-dire qu'il doit s'agir d'une expression au référent identifiable pour l'auditeur / lecteur. Dans les langues qui, comme le japonais, ont un marqueur de topicalité, celui-ci ne peut apparaître qu'avec un SN dont le référent est défini ou générique; de même, la dislocation à droite, structure généralement associée au marquage du topique, n'autorise que des SN à référence définie ou générique (cf. Gundel 1985 : 91).

${ }^{12}$ Fréquemment dans des structures elliptiques, faisant l'économie de l'auxiliaire (3a) ou du verbe attributif être (3b).

${ }^{13}$ Cette structure fait partie du type «parataxe» dans la typologie de Sullet-Nylander (1998). En plus du type de titres illustré par (5)-(6), le type parataxe comprend des structures appositives telles que Ri Sol-ju, première dame coréenne (Libération, 26 juillet 2012) ou de juxtaposition coordonnante comme dans Madonna, ô Graal, ô désespoir (Libération, 26 juillet 2012 : exemple dans lequel on pourrait remplacer la virgule après Madonna par la conjontion $o u)$, types de titres qu'on n'évoquera pas ici.

${ }^{14}$ Dans ce dernier cas, on peut trouver une virgule à la place des deux points : JO, Londres triple vainqueur (Le Monde, 26 juillet 2012), PSA-Montebourg, beaucoup de cris pour rien (Libération, 26 juillet 2012).

${ }^{15}$ Fonction de pseudo-rubrique selon Bosredon \& Tamba (1992: 42), qui proposent eux-mêmes une glose en $\grave{a}$ propos de ou en ce qui concerne.

${ }^{16}$ Pas dans tous : certains titres constituent des reprises de titres de la première page ; lors de leur nouvelle mention, les référents peuvent être considérés comme semi-actifs (Lambrecht 1994: 99-100), plus précisément comme « accessibles textuellement » (Lambrecht 1994, 100, parle de référents « accessibles textuellement » dans les cas de désactivation d'un état antérieur actif dans le discours). C'est le cas, par exemple, du référent associé au SN initial du titre Honoraires libres : pourquoi les médecins résistent (Le Monde, 26 juillet 2012, p.7), forme de reprise du SN initial du titre antérieur Dépassements d'honoraires : les médecins sur la défensive (Le Monde, 26 juillet 2012, p.1).
} 